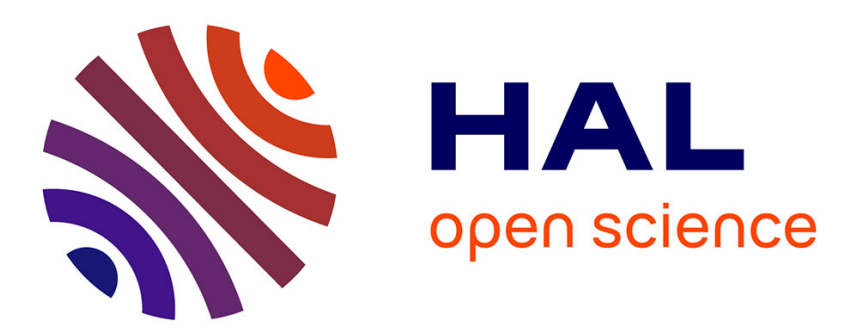

\title{
On the complexity of Independent Dominating Set with Obligations in graphs
}

Christian Laforest, Timothée Martinod

\section{To cite this version:}

Christian Laforest, Timothée Martinod. On the complexity of Independent Dominating Set with Obligations in graphs. [Research Report] LIMOS (UMR CNRS 6158), université Clermont Auvergne, France. 2020. hal-02946979v2

\section{HAL Id: hal-02946979 \\ https://hal.science/hal-02946979v2}

Submitted on 9 Jan 2021

HAL is a multi-disciplinary open access archive for the deposit and dissemination of scientific research documents, whether they are published or not. The documents may come from teaching and research institutions in France or abroad, or from public or private research centers.
L'archive ouverte pluridisciplinaire HAL, est destinée au dépôt et à la diffusion de documents scientifiques de niveau recherche, publiés ou non, émanant des établissements d'enseignement et de recherche français ou étrangers, des laboratoires publics ou privés. 


\title{
On the complexity of Independent Dominating Set with Obligations in graphs
}

\author{
Christian Laforest, Timothée Martinod ${ }^{1}$ \\ Laboratoire d'Informatique, de Modélisation et d'Optimisation des Systèmes, Université Clermont \\ Auvergne, CNRS UMR 6158, 63178 Aubière, FRANCE
}

\begin{abstract}
A subset $D \subseteq V$ is an independent (or stable) dominating set of a graph $G=(V, E)$ if $D$ is an independent set (no edges between vertices of $D$ ) and dominates all the vertices of $G$ (each vertex of $V-D$ has a neighbour in $D$ ). In this paper we study a generalisation of this classical notion. Namely, an instance of our problem is a graph $G=(V, E)$ and $\Pi=\left(V_{1}, \ldots, V_{k}\right)$ a partition of $V$. Each subset $V_{i}$ of $\Pi$ is called an obligation. An Independent Dominating set with Obligation (IDO) $D$ in an instance $(G, \Pi)$ is an independent dominating set of $G$ with the additional property that if a vertex $u$ of obligation $V_{i}$ is in $D$, then all the others vertices of $V_{i}$ must also be in $D$; i.e., for all $i=1, \ldots, k$, either $V_{i} \cap D=\emptyset$ or $V_{i} \subseteq D$ (we say that $D$ respects the obligations). Note that when each obligation of $\Pi$ is a singleton, an IDO is just an independent dominating set of $G$ that can be constructed with a greedy algorithm. Among other things, we show that determining if an instance $(G=(V, E), \Pi)$ has an $I D O$ is $N P$-complete, even if $G$ is a path (each vertex of $G$ exactly has two neighbours, except two extremities that have one), all the obligations are independent sets of $G$ and they all have the same constant size $\lambda>1$. We also show that the problem is also $N P$-complete, even if $\Pi$ is composed of $\sqrt{|V|}$ independent obligations each of size $\sqrt{|V|}$ or if the diameter of $G$ is three. Our results clearly show that this problem is very difficult, even in extremely restricted instances. Hence, in a second part of the paper, we relax the condition to dominate all the vertices of $G$. However, we show that determining if $(G=(V, E), \Pi)$ contains an independent set $D$ respecting the obligations and dominating at least $3 \sqrt{|V|}-2$ vertices of $G$ is $N P$-complete, even if $G$ is a collection of disjoint paths and obligations are all independent sets of $G$. On the positive side, we propose a greedy algorithm constructing a solution dominating at least $2 \sqrt{|V|}-1$ vertices in any instance $(G=(V, E)$, $\Pi)$ if all obligations of $\Pi$ are independent sets of $G$.
\end{abstract}

This work was sponsored by a public grant overseen by the French National Research Agency as part of the "Investissements d'Avenir" through the IMobS3 Laboratory of Excellence (ANR-10-LABX-0016) and the IDEX-ISITE initiative CAP 20-25 (ANR-16-IDEX-0001).

${ }^{1}$ Corresponding author

Email addresses: christian.laforest@uca.fr (Christian Laforest), timothee.martinod@uca.fr (Timothée Martinod) 
Keywords: Domination; NP-completeness

\section{Introduction}

Let $G=(V, E)$ be an undirected graph, with $V$ its set of vertices and $E$ its set of edges. Let $D \subseteq V$ be a subset of vertices. Set $D$ is a stable set or independent set of $G$ if $G$ contains no edge between vertices of $D$. Set $D$ is a dominating set of $G$ if each vertex of $V-D$ has at least one neighbour in $D$. Set $D$ is an independent dominating set of $G$ if $D$ is an independent set and a dominating set of $G$.

There are many variants of domination problems according to the additional properties that are considered, namely the independent dominating set, the total dominating set, the connected dominating set, among others (see [9] and [8]). These problems are central in graph theory. They are also useful and can be used in distributed applications where the goal is to install services or devices on some machines/vertices such that every vertex is neighbour of at least one vertex having the device.

It is well-known that any graph contains at least an independent dominating set. From an algorithmic point-of-view, given a graph $G=(V, E)$ and an integer $k$, determining if $G$ contains an independent dominating set of size at most $k$ is $N P$-complete (see [7]). The complexity of the best known exact algorithm to construct a minimum size dominating set is $O\left(1.4969^{|V|}\right)$, with a measure and conquer approach (see [17]). The problem of independent dominating set was first formalized by Berge [2] and Ore [16] in 1962 and 1965. Garey has shown that finding the smallest independent dominating set of a graph is NP-complete [7]. Independent dominating set has been studied in the literature in the lasts decades. In 1991 Irving [10] has shown that, unless $P=N P$, no polynomialtime approximation algorithm for this problem can guarantee to find a minimum size independent dominating set within a factor of $K$, where $K>1$ is any fixed constant, even in bipartite graphs. In 2004 Chlebík and Chlebíková [3] have shown that it is NPhard to approximate the minimum size of an independent dominating set in a graph of maximal degree three within a $1+1 / 390$ ratio even in bipartite graphs. In 2020 Akbari et al. [1] have provided a family of connected cubic graphs of $n$ vertices where the minimum size of an independent dominating set is $3 n / 8$.

In this paper, we study the independent dominating set problem with additional constraints. In our version, an instance contains a graph $G=(V, E)$ and a partition $\Pi=\left(V_{1}, \ldots, V_{k}\right)$ of the set $V$ of vertices of $G$ (i.e. $V_{i} \subseteq V$ for all $i=1, \ldots, k, V_{i} \cap V_{j}=\emptyset$ for all $i \neq j$ and $\left.\cup_{i=1}^{k} V_{i}=V\right)$. Each subset $V_{i}$ of $\Pi$ is called an obligation in our paper. Now, given any instance $\left(G=(V, E), \Pi=\left(V_{1}, \ldots, V_{k}\right)\right)$, an independent dominating set with obligation (IDO) $D$ is an independent dominating set of $G$, respecting the obligations of $\Pi$, that is: for all $i=1, \ldots, k$, either $V_{i} \subseteq D$ or $V_{i} \cap D=\emptyset$. Note that when all the obligations are singletons, any $I D O$ is just an independent dominating set of $G$. 
An obligation can represent a subset of devices that must be used together, not individually, or a team of people that must all be present to make an action. Obligations were recently introduced in [5] for many graph problems such as vertex cover, connected vertex cover, dominating set, total dominating set, independent dominating set, spanning tree, matching and hamiltonian path. The authors mainly obtained hardness results. Studying classical graph problems with additional constraints is not new. The introduction of obligations as an object of study was motivated by considering a sort of "reverse" constraint of conflicts. They have been studied during the last years. The general context of conflict constraint is the following. Given a graph $G=(V, E)$, we add a set of pairs of elements of $G$ (vertices or edges) that cannot both be in a same solution (that can be a path, a tree, a dominating set, etc. depending of the goal). At the opposite of obligations, a conflict models the fact that two elements of a system cannot be used simultaneously, for example because they are incompatible. Most problems with conflicts are hard, even if the underlying classical version is polynomial. See for example the following recent publications on the subject: $[4,6,11,12,13,14,15]$.

If all the obligations of $\Pi$ have the same size $\lambda$, we say that they are balanced or $\lambda$-balanced. If an obligation $V_{i} \in \Pi$ is an independent set of $G$, it is called stable (or independent). Note that if an obligation is not stable, it cannot be part of an IDO; if all the obligations are not stable then $(G, \Pi)$ does not contain any IDO. Hence, in our generalisation, some instances may not contain any IDO. For that reason we mainly focus our attention on instances where obligations are all independent.

\section{IDO Problem:}

Instance: $(G, \Pi)$ where $G=(V, E)$ is an undirected graph and $\Pi=V_{1}, \ldots, V_{k}$ a partition of $V$.

Question: Does $(G, \Pi)$ contain an $I D O$ ?

The IDO problem was first introduced by Cornet and Laforest in [5]. They proved its NP-completeness even with obligations of maximal size two. However, they used non-stable obligations for the reduction. Because of the previously made remark, it is legitimate to investigate the problem with instances composed of only stable obligations.

In this paper we improve the knowledge of this problem. We prove that the IDO problem is $N P$-complete in different cases according to the topology of the graph, the stability and the balance of the obligations. In the whole paper a path is a connected graph in which each vertex has exactly two neighbours, except two extremities that have only one. A graph is a collection of disjoint paths if it is only composed of paths that are pairwise vertex-disjoint (it is a forest of paths). Thus a path is a very simple structured connected graph with low degree. It has the properties of being a tree, being bipartite and planar. Most algorithmic problems are trivial or easy to solve in paths. However, we show in Section 2 (Theorem 3) that the IDO problem is $N P$-Complete, even if $G$ is a path and all the obligations are independent and $\lambda$-balanced for any constant $\lambda \geq 2$ (N.B. if the obligations are 1-balanced, the IDO problem is polynomial). The reduction involves many intermediate constructions that are presented step by step and illustrated by several figures. We also show in Theorem 4 that even if the graph has diameter three the $I D O$ problem remains $N P$-complete. 
As mentioned previously, even if the size of obligations is constant the problem is $N P$-complete. Thus we turn our attention on non-constant ones in Section 3. However, we show in Theorem 5 that the $I D O$ problem is $N P$-complete even in instances $(G=$ $(V, E), \Pi)$ where obligations are independent and $\sqrt{|V|}$-balanced. Still in Section 3 we prove several other $N P$-completeness results with other types of comparisons between the sizes and the number of obligations.

Due to all these hardness results, in Section 4 we relax the constraint of dominating all the vertices of the graph. To do that we introduce a maximisation version. We called this the Partially Independent Dominating set with Obligation (PIDO) problem. We show that deciding if an instance $(G=(V, E), \Pi)$ contains an independent set $D$ of $G=(V, E)$, respecting the obligations of $\Pi$ and dominating at least $3 \sqrt{|V|}-2$ vertices of $G$ is $N P$ complete, even if $G$ is a collection of disjoint paths and obligations are all independent. On the positive side, we propose a polynomial time algorithm constructing a solution in

any instance $(G=(V, E), \Pi)$, dominating at least $2 \sqrt{|V|}-1$ vertices if obligations are all independent. In Section 5 we conclude this paper.

As many results in this paper are obtained by a reduction from the $N P$-complete Restricted Exact Cover by 3-Sets problem (see [7]) we describe it here. Let $\mathcal{X}$ be a finite set of elements and $\mathcal{C}$ a collection of triplets (sets of three elements) of $\mathcal{X}$. Each element of $\mathcal{X}$ appears in exactly three subsets of $\mathcal{C}$; thus there are $3 q$ elements and $3 q$ triplets. Given such an instance $(\mathcal{X}, \mathcal{C})$ it is $N P$-complete to decide if there is a sub-collection $C^{\prime} \subseteq \mathcal{C}$ such that each element of $\mathcal{X}$ occurs in exactly one triplet of $C^{\prime}$. Note that if it exists, such a sub-collection $C^{\prime}$ has exactly $q$ disjoint triplets.

\section{$2 \quad I D O$ in a path}

In this section, we show that the IDO problem is $N P$-complete even in a path with stable and $\lambda$-balanced obligations for any constant $\lambda \geq 2$. As said above, we will do a reduction from the $R X 3 C$ problem. Since the construction uses several gadgets, we will proceed step by step. First, each element in $R X 3 C$ is in exactly three triplets. To represent membership of the elements and the intersection between triplets, we will create one gadget per element.

Construction 1. Let $(\mathcal{X}, \mathcal{C})$ be an instance of $R X 3 C$ where $\mathcal{X}=\left\{x_{1}, \ldots, x_{3 q}\right\}$ and $\mathcal{C}=$ $\left\{c_{1}, \ldots, c_{3 q}\right\}$. For each element $x_{i} \in \mathcal{X}$ we construct an associated gadget composed of a graph and some obligations.

- Let $c_{j}, c_{k}, c_{l} \in \mathcal{C}$ with $j<k<l$ be the three triplets containing $x_{i}$.

- We add 16 obligations denoted $y_{i}^{T}, y_{i}^{F}, y_{i}^{1}, \ldots, y_{i}^{11}, x_{i}^{1}, x_{i}^{2}$ and $x_{i}^{3}$ each composed of two independent vertices. In each obligation $y$ and $x$ we consider one left vertex and one right vertex respectively denoted by $L(y)$ and $R(y)$ or $L(x)$ and $R(x)$. 
- We add the vertices $Z_{j}, Z_{k}$ and $Z_{l}$ that are vertices of gadgets (defined later) representing the triplets containing element $x_{i}$.

- We add the edges $\left(R\left(y_{i}^{T}\right) L\left(y_{i}^{F}\right)\right),\left(R\left(y_{i}^{F}\right) L\left(y_{i}^{1}\right)\right),\left(R\left(y_{i}^{F}\right) L\left(y_{i}^{2}\right)\right),\left(L\left(y_{i}^{1}\right) R\left(y_{i}^{4}\right)\right),\left(R\left(y_{i}^{1}\right) L\left(y_{i}^{4}\right)\right)$, $\left(R\left(y_{i}^{1}\right) R\left(y_{i}^{2}\right)\right),\left(L\left(y_{i}^{2}\right) L\left(y_{i}^{3}\right)\right),\left(R\left(y_{i}^{3}\right) L\left(y_{i}^{5}\right)\right),\left(R\left(y_{i}^{3}\right) L\left(y_{i}^{6}\right)\right)$, $\left(R\left(y_{i}^{4}\right) L\left(y_{i}^{9}\right)\right),\left(L\left(y_{i}^{5}\right) R\left(y_{i}^{7}\right)\right),\left(R\left(y_{i}^{5}\right) R\left(y_{i}^{6}\right)\right),\left(R\left(y_{i}^{5}\right) L\left(y_{i}^{7}\right)\right),\left(L\left(y_{i}^{6}\right) R\left(y_{i}^{8}\right)\right)$, $\left(R\left(y_{i}^{6}\right) L\left(y_{i}^{8}\right)\right),\left(R\left(y_{i}^{7}\right) L\left(y_{i}^{10}\right)\right),\left(R\left(y_{i}^{8}\right) L\left(y_{i}^{11}\right)\right),\left(R\left(y_{i}^{9}\right) L\left(x_{i}^{1}\right)\right),\left(R\left(y_{i}^{10}\right) L\left(x_{i}^{2}\right)\right)$, $\left(R\left(y_{i}^{11}\right) L\left(x_{i}^{3}\right)\right),\left(R\left(x_{i}^{1}\right) Z_{j}\right),\left(R\left(x_{i}^{2}\right) Z_{k}\right)$ and $\left(R\left(x_{i}^{3}\right) Z_{l}\right)$.

Note that the gadget associated to each element $x_{i}$ is composed of a graph that is a collection of disjoint paths, and 16 stable 2-balanced (if we exclude the $Z$-vertices) obligations. The aggregation of these $3 q$ gadgets has $48 q$ obligations. See an illustration in Figure 1. Six vertices are represented differently for clarity purposes. The green squares and the orange polygons are the interface vertices of the link between triplets and their three elements. Each gadget of element is composed of the obligation $y, x$ and the subgraph induced by their vertices. Each gadget of element is connected to exactly three gadgets of triplet (defined later, see an illustration in Figure 2). This representation will also appear in Figure 2 and Figure 3.

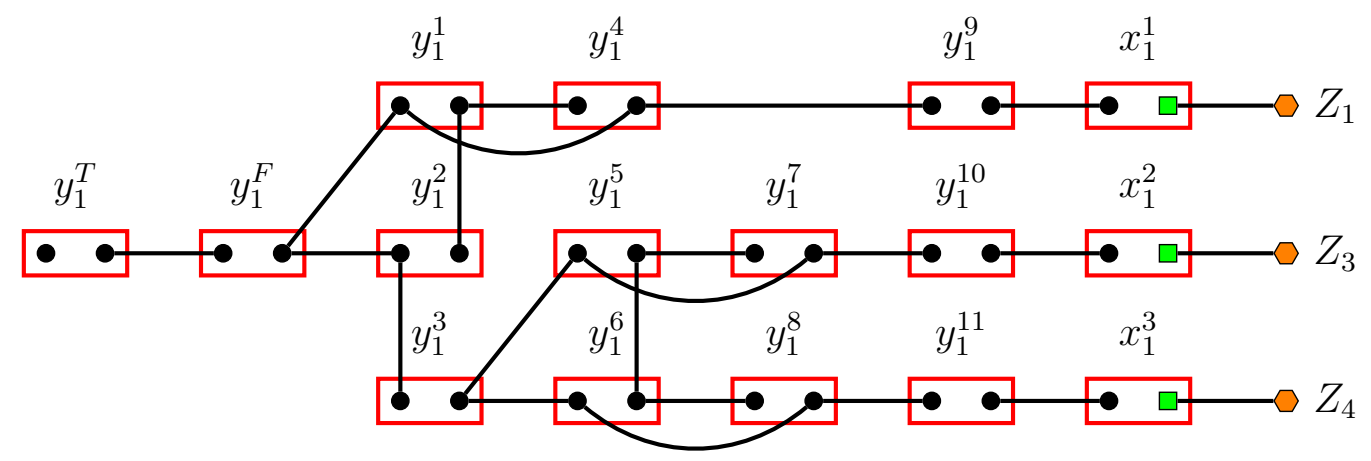

Figure 1: Illustration of Construction 1 only for the element 1 of the instance $(\mathcal{X}, \mathcal{C})$ of $R X 3 C$ where $\mathcal{X}=\{1, \ldots, 6\}$ and $\mathcal{C}=\{\{1,2,3\},\{2,3,4\},\{1,2,6\},\{1,5,6\},\{3,4,5\}$, $\{4,5,6\}\}$.

Lemma 1. Let $(\mathcal{X}, \mathcal{C})$ be an instance of $R X 3 C$. Let $H$ be the gadget of the element $x_{i}$ following Construction 1 from $(\mathcal{X}, \mathcal{C})$. Gadget $H$ does not contain an IDO having neither $Z_{j}, Z_{k}$ nor $Z_{l}$.

Proof. Suppose that $H$ contains an IDO $S$ which has neither $Z_{j}, Z_{k}$ nor $Z_{l}$. Therefore obligations $x_{i}^{1}, x_{i}^{2}$ and $x_{i}^{3}$ must be in $S$ and so must be $y_{i}^{4}, y_{i}^{7}, y_{i}^{8}$ and $y_{i}^{3}$. Since $y_{i}^{T}$ has an isolated vertex, $y_{i}^{T}$ is in $S$ and the right vertex of $y_{i}^{F}$ has to be dominated by $y_{i}^{1}$ or $y_{i}^{2}$. However, they cannot be in $S$ because of the two edges $\left(R\left(y_{i}^{1}\right) L\left(y_{i}^{4}\right)\right)$ and $\left(L\left(y_{i}^{2}\right) L\left(y_{i}^{3}\right)\right)$, which is a contradiction.

The next result is easy to verify. 
Lemma 2. Let $(\mathcal{X}, \mathcal{C})$ be an instance of $R X 3 C$. Let $H$ be the gadget of any element $x_{i} \in \mathcal{C}$ following Construction 1 from $(\mathcal{X}, \mathcal{C})$. Let $S_{j}$ be the set of vertices composed of vertex $Z_{j}$ plus vertices of obligations $y_{i}^{9}, y_{i}^{1}, y_{i}^{3}, y_{i}^{7}, y_{i}^{8}, x_{i}^{2}, x_{i}^{3}, y_{i}^{T}$. Let $S_{k}$ be the set composed of vertex $Z_{k}$ plus vertices of obligations $y_{i}^{10}, y_{i}^{5}, y_{i}^{8}, y_{i}^{2}, y_{i}^{4}, x_{i}^{1}, x_{i}^{3}, y_{i}^{T}$. Let $S_{l}$ be the set composed of vertex $Z_{l}$ plus vertices of obligations $y_{i}^{11}, y_{i}^{6}, y_{i}^{7}, y_{i}^{2}, y_{i}^{4}, x_{i}^{1}, x_{i}^{2}, y_{i}^{T}$. These three sets are IDO of $H$ containing only one vertex of $\left\{Z_{j}, Z_{k}, Z_{l}\right\}$

Lemma 3. Let $(\mathcal{X}, \mathcal{C})$ be an instance of $R X 3 C$. Let $H$ be the gadget of any element $x_{i}$ following Construction 1 from $(\mathcal{X}, \mathcal{C})$. Gadget $H$ contains no IDO having more than one vertex among $Z_{j}, Z_{k}$ and $Z_{l}$.

Proof. Suppose there is a solution $S$ containing $Z_{k}$ and $Z_{l}$. Since $Z_{k}$ and $Z_{l}$ are in $S$, $y_{i}^{10}$ and $y_{i}^{11}$ must be in $S$. Therefore $S$ must contain $y_{i}^{5}$ and $y_{i}^{6}$ which is a contradiction because of the edge between $R\left(y_{i}^{5}\right)$ and $R\left(y_{i}^{6}\right)$.

Suppose there is a solution $S$ containing $Z_{j}$ and one vertex among $Z_{k}$ and $Z_{l}$. Since $Z_{k} \in S$ or $Z_{l} \in S$, either $y_{i}^{10}, y_{i}^{5}$ and $y_{i}^{2}$ are in $S$ or $y_{i}^{11}, y_{i}^{6}$ and $y_{i}^{2}$ are in $S$. Since $Z_{j} \in S$, $y_{i}^{9}$ and $y_{i}^{1}$ must be in $S$, which is a contradiction because of the edge between $R\left(y_{i}^{1}\right)$ and $R\left(y_{i}^{2}\right)$.

We can conclude that each gadget representing an element $x_{i}$ admits a solution for the IDO problem if and only if there is exactly one vertex $Z$ in the solution (that will represent the triplet which contains $x_{i}$ ). Now we add a gadget per triplet of $\mathcal{C}$ and we combine them with all the gadgets of elements defined in Construction 1.

Construction 2. Let $(\mathcal{X}, \mathcal{C})$ be an instance of $R X 3 C$ where $\mathcal{X}=\left\{x_{1}, \ldots, x_{3 q}\right\}$ and $\mathcal{C}=$ $\left\{c_{1}, \ldots, c_{3 q}\right\}$. We construct an instance $(G=(V, E), \Pi)$ of the IDO problem as follows.

- We use Construction 1 to construct a gadget for each element of $\mathcal{X}$.

- We construct now a gadget for each triplet of $\mathcal{C}$. Let us note $Z_{i}^{a}, Z_{i}^{b}, Z_{i}^{d}$ the three vertices representing a triplet $c_{i} \in \mathcal{C}$ as they appear in the three gadgets of the three elements that $c_{i}$ contains.

- We add three 2-balanced obligations $z_{i}^{a}$, $z_{i}^{b}$ and $z_{i}^{d}$ which contain respectively vertices $Z_{i}^{a}, Z_{i}^{b}, Z_{i}^{d}$ and a new vertex in each, respectively denoted $R\left(z_{i}^{a}\right), R\left(z_{i}^{b}\right)$ and $R\left(z_{i}^{d}\right)$.

- We add three 2-balanced obligations $w_{i}^{1}, w_{i}^{2}$ and $w_{i}^{3}$ composed of two independent vertices. In each obligation $w$ we consider one left vertex and one right vertex respectively represented by $L(y)$ and $R(y)$.

- We add the edges $\left(R\left(z_{i}^{a}\right) L\left(w_{i}^{1}\right)\right),\left(R\left(z_{i}^{b}\right) L\left(w_{i}^{1}\right)\right),\left(R\left(z_{i}^{d}\right) L\left(w_{i}^{3}\right)\right),\left(R\left(w_{i}^{1}\right) L\left(w_{i}^{2}\right)\right)$ and $\left(R\left(w_{i}^{2}\right) R\left(w_{i}^{3}\right)\right)$.

Note that $G$ is a collection of disjoint paths, and $\Pi$ has $66 q$ stable 2-balanced obligations. Figure 2 shows a gadget associated to a triplet of $\mathcal{C}$. Figure 3 represents the "big picture" of the overall construction; each ellipse represents the gadget of an element of $\mathcal{X}$, and each pentagon represents the gadget of a triplet of $\mathcal{C}$. 


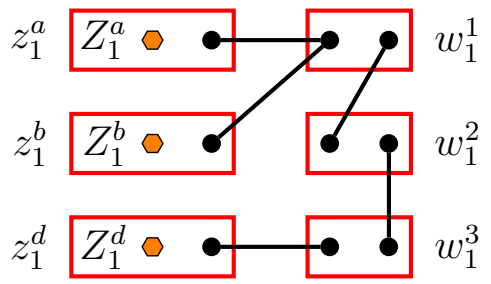

Figure 2: Illustration of Construction 2 for the triplet $c_{1}$ of the instance $(\mathcal{X}, \mathcal{C})$ of $R X 3 C$ where $\mathcal{X}=\{1, \ldots, 6\}$ and $\mathcal{C}=\{\{1,2,3\},\{2,3,4\},\{1,2,6\},\{1,5,6\},\{3,4,5\},\{4,5,6\}\}$.

Lemma 4. Let $(\mathcal{X}, \mathcal{C})$ be an instance of $R X 3 C$. Let $(G, \Pi)$ be the instance obtained by Construction 2 from $(\mathcal{X}, \mathcal{C})$. Suppose that $(G, \Pi)$ has an IDO $S$. Let $H$ be the gadget associated to a triplet $c_{i}$ in $(G, \Pi)$. Either $S$ contains the three obligations $z_{i}^{a}, z_{i}^{b}$ and $z_{i}^{d}$ of $H$ or none of them.

Proof. Suppose first that $S$ contains the obligation $w_{i}^{2}$. Then obligations $w_{i}^{1}$ and $w_{i}^{3}$ cannot be in $S$. But $S$ still has one vertex to dominate in each. Therefore the obligations $z_{i}^{a}$ and $z_{i}^{d}$ must be in $S$. Since $L\left(w_{i}^{1}\right)$ cannot dominate $R\left(z_{i}^{b}\right)$, the obligation $z_{i}^{d}$ must be in $S$. Thus, all three $z_{i}$ obligations are in $S$.

Suppose now that the obligation $w_{i}^{2}$ is not in $S$. The obligations $w_{i}^{1}$ and $w_{i}^{3}$ must be in $S$. Thus, no $z_{i}$ obligations can be in $S$.

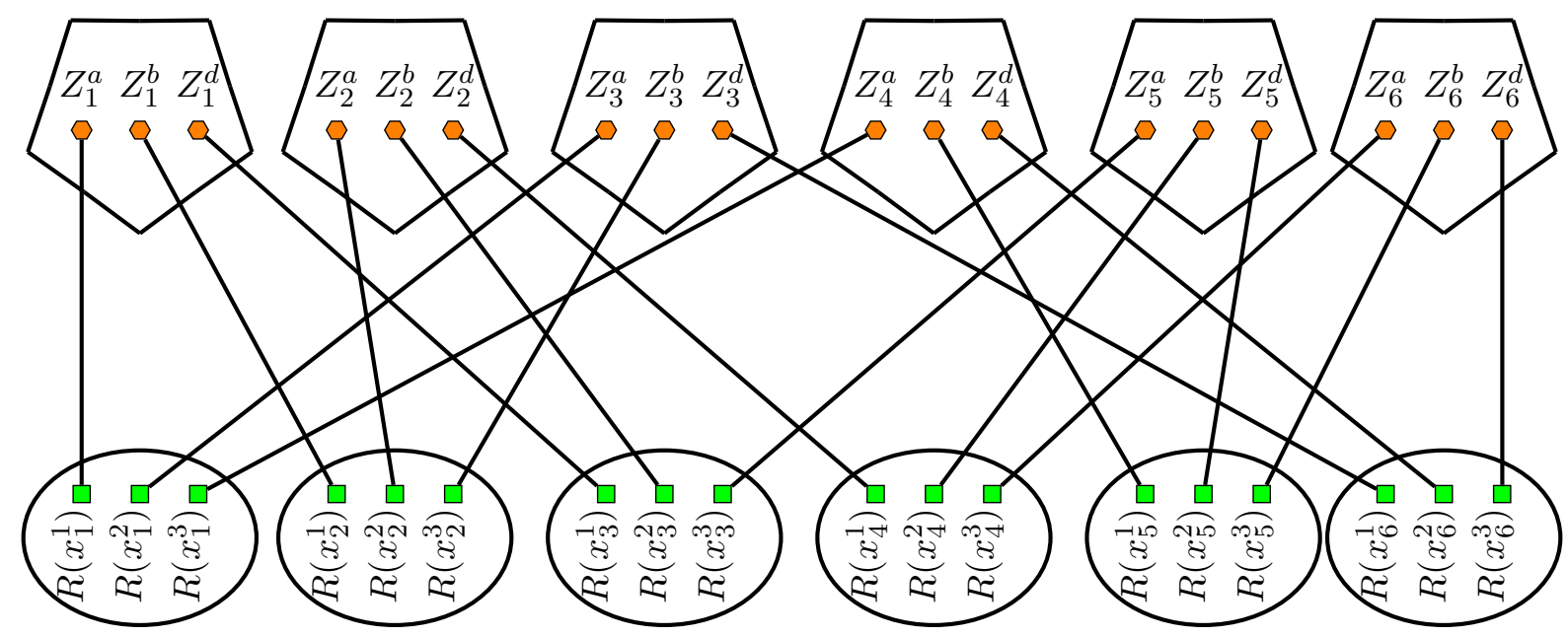

Figure 3: Illustration of Construction 2 for the instance $(\mathcal{X}, \mathcal{C})$ of $R X 3 C$ with $\mathcal{X}=\{1, \ldots, 6\}$ and $\mathcal{C}=\{\{1,2,3\},\{2,3,4\},\{1,2,6\},\{1,5,6\},\{3,4,5\},\{4,5,6\}\}$. Pentagons represent gadgets like in Figure 2. Ellipses represent gadgets like the ones in Figure 1.

Theorem 1. The IDO problem is NP-complete, even if the graph is a collection of disjoint paths and the obligations are stable and 2-balanced. 
Proof. The problem is clearly in NP. Moreover, constructing $(G, \Pi)$ from an $R X 3 C$ instance $(\mathcal{X}, \mathcal{C})$ is polynomial.

Suppose there is a solution $S$ for the instance $(\mathcal{X}, \mathcal{C})$ of $R X 3 C$. Let us construct an $I D O S^{\prime}$ for $(G, \Pi)$. For each $c_{i} \in S$ we put the obligations $z_{i}^{a}, z_{i}^{b}, z_{i}^{d}$ and $w_{i}^{2}$ in $S^{\prime}$. For each $c_{i} \notin S$ we put the obligations $w_{i}^{1}$ and $w_{i}^{3}$ in $S^{\prime}$. For each $Z_{i} \in S^{\prime}$, we add in $S^{\prime}$ the well-chosen set given by Lemma 2 containing vertex $Z_{i}$ for each gadget of element contained in triplet $c_{i}$. The set $S^{\prime}$ respects the obligations of $\Pi$, is a stable set of $G$, and dominates all the vertices of $G$ (thanks to the fact that $S$ is an exact cover of $\mathcal{X}$ ), thus is an $I D O$ of $(G, \Pi)$.

Suppose now there is an $I D O S$ in the instance $(G, \Pi)$. Let us construct a solution $S^{\prime}$ for the $R X 3 C$ problem. For each $Z_{i} \in S$ put triplet $c_{i}$ in $S^{\prime}$. By Lemma 1, each element of $\mathcal{X}$ is covered by at least one triplet of $S^{\prime}$ and by Lemma 3 each element is covered by at most one triplet. Hence $S^{\prime}$ is a solution for the $R X 3 C$ problem.

In what follows we extend the result and constructions for Theorem 1 to instances having $\lambda$-balanced obligations where $\lambda \geq 2$ is any constant integer. The general idea is the following (details are given later). If $\lambda$ is even, we duplicate $\frac{\lambda}{2}$ times the instance (graph and obligations) of Construction 2 and then we merge the copies. If $\lambda$ is odd we add one vertex per obligation (and some edges) of each gadget of element or gadget of triplet, while keeping all the good properties.

Let us start with the case where $\lambda$ is even. Take $k=\frac{\lambda}{2}$ disjoint identical copies, noted $I_{1}=\left(G_{1}, \Pi_{1}\right), \ldots, I_{k}=\left(G_{k}, \Pi_{k}\right)$ of Construction 2. Now, construct a new IDO instance $(G, \Pi)$ in which the graph $G=(V, E)$ is composed of the union of these $k$ graphs: $V=V_{1} \cup \cdots \cup V_{k}$ and $E=E_{1} \cup \cdots \cup E_{k}$. As each $G_{i}$ is a collection of disjoint paths, this is also the case for $G$ ( $G$ is composed of "parallel" copies of the "same" graph). Now we merge the obligations. Let $B$ be any obligation of $\Pi_{1}$. As the $k$ instances are identical, a similar obligation $B$ is also part of each other instance. Now, all the vertices of these $k$ similar obligations $B$ are grouped in a new obligation of $\Pi$. As $|B|=2$, this new obligation, that we also call $B$, of $\Pi$ is of size $\lambda$. It is also an independent set of graph $G$. As the $k$ copies of graphs are disjoint in $G$ and as identical obligations are merged, the overall instance $(G, \Pi)$ has an $I D O$ if and only if each $\left(G_{i}, \Pi_{i}\right) i=1, \ldots, k$ has an $I D O$.

Let us now study the case where $\lambda$ is odd. Let us start with $\lambda=3$.

Construction 3. Let $(\mathcal{X}, \mathcal{C})$ be an instance of $R X 3 C$ where $\mathcal{X}=\left\{x_{1}, \ldots, x_{3 q}\right\}$ and $\mathcal{C}=$ $\left\{c_{1}, \ldots, c_{3 q}\right\}$. We construct a new instance $(G=(V, E), \Pi)$ of the IDO problem as follows:

- We follow Construction 2.

- For each $x_{i} \in \mathcal{X}$ :

- In each obligation $y_{i}$ and $x_{i}$ we add a new vertex denoted respectively $N\left(y_{i}\right)$ and $N\left(x_{i}\right)$. 
- We add the edges $\left(N\left(y_{i}^{T}\right) N\left(y_{i}^{F}\right)\right),\left(N\left(y_{i}^{1}\right) N\left(y_{i}^{2}\right)\right),\left(N\left(y_{i}^{1}\right) N\left(y_{i}^{4}\right)\right),\left(N\left(y_{i}^{3}\right) N\left(y_{i}^{5}\right)\right)$, $\left(N\left(y_{i}^{3}\right) N\left(y_{i}^{6}\right)\right),\left(N\left(y_{i}^{5}\right) N\left(y_{i}^{7}\right)\right),\left(N\left(y_{i}^{6}\right) N\left(y_{i}^{8}\right)\right), \quad\left(N\left(y_{i}^{9}\right) N\left(x_{i}^{1}\right)\right),\left(N\left(y_{i}^{10}\right) N\left(x_{i}^{2}\right)\right)$ and $\left(N\left(y_{i}^{11}\right) N\left(x_{i}^{3}\right)\right)$.

- For each $c_{i} \in \mathcal{C}$ :

- In each obligation $w_{i}$ and $z_{i}$ we add a new vertex denoted respectively $N\left(w_{i}\right)$ and $N\left(z_{i}\right)$.

- We add the edges $\left(N\left(w_{i}^{1}\right) N\left(z_{i}^{a}\right)\right),\left(N\left(w_{i}^{1}\right) N\left(z_{i}^{b}\right)\right),\left(N\left(w_{i}^{3}\right) N\left(z_{i}^{d}\right)\right)$ and $\left(N\left(w_{i}^{3}\right) N\left(w_{i}^{2}\right)\right)$.

Note that $G$ is a collection of disjoint paths, and $\Pi$ contains $66 q$ stable 3-balanced obligations. See two illustrations in Figure 4 and Figure 5. The obligations represented in Figure 4 and Figure 5 are the same than the ones in Figure 1 and Figure 2, but only the new vertices and new edges added by Construction 3 are shown. In other words, all the obligations have both vertices of Construction 1 (respectively Construction 2) and vertices of Construction 3.

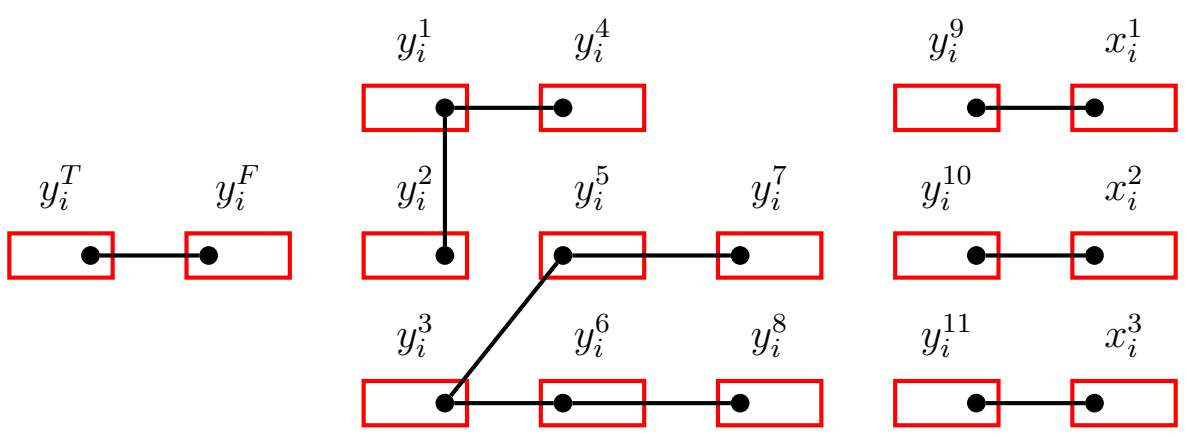

Figure 4: Illustration of Construction 3 only for an element $i$ of the instance $(\mathcal{X}, \mathcal{C})$ of $R X 3 C$.

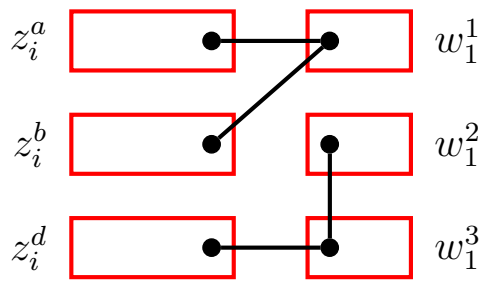

Figure 5: Illustration of Construction 3 only for a triplet $c_{i}$ of the instance $(\mathcal{X}, \mathcal{C})$ of $R X 3 C$.

Before proceeding to obligations with more than three vertices, let us see an $I D O$ with obligations of size three. All the obligations have three vertices (two original vertices and one new vertex) after Construction 3. Since no edge connects the new vertices and the original vertices, an IDO in this instance can be considered as two (dependent) IDO $S_{1}$ 
and $S_{2}$. One $\left(S_{1}\right)$ dominates the original vertices, and another $\left(S_{2}\right)$ dominates the new vertices. The part of the IDO which dominates the original vertices $\left(S_{1}\right)$ still admits only three solutions in each gadget of element, and each representing vertices of triplets are either all in an IDO or none are in an IDO. The other part of the IDO $\left(S_{2}\right)$ has to respect the obligations chosen in $S_{1}$. This constraint leads to the existence of a unique IDO in the new part for an IDO in the original part. For each chosen IDO $S_{1}$, all the new vertices are already dominated. The obligations in the IDO remain the same despite the new vertex in each obligation. Thanks to this constraint, each instance still admits only one triplet for each element, and either all the representing of a triplet are in an IDO or none.

Let us proceed to the construction of an instance of $I D O$ with more than three vertices in each obligation.

Construction 4. Let $(\mathcal{X}, \mathcal{C})$ be an instance of $R X 3 C$ where $\mathcal{X}=\left\{x_{1}, \ldots, x_{3 q}\right\}$ and $\mathcal{C}=$ $\left\{c_{1}, \ldots, c_{3 q}\right\}$. Let $\lambda \geq 2$ be an integer. We construct $G=(V, E)$ a graph and obligations $\Pi$ as follows:

- If $\lambda$ is even: we duplicate Construction $2 \frac{\lambda}{2}$ times and merge the copies.

- If $\lambda$ is odd:

- We duplicate Construction $2 \frac{\lambda-1}{2}$ times and we merge the copies.

- In the previous instance, we add new vertices and new edges in gadgets of elements and triplets following the structure presented in Figure 4 and Figure 5.

Now $G$ is a collection of disjoint paths and $\Pi$ contains stable $\lambda$-balanced obligations. Since Construction 4 constructs a constant number of "parallel" and "independent" copies of the initial one given by Construction 2 used to prove Theorem 1, we can use it to get the following more general result.

Theorem 2. The IDO problem is NP-complete, even if the graph is a collection of disjoint paths and the obligations are stable and $\lambda$-balanced where $\lambda \geq 2$ is a constant integer.

Theorem 2 states $N P$-completeness when the graph is a collection of disjoint paths. In the following, we use a new gadget, called the neutral connector, to connect all these disjoint paths and to prove the NP-completeness when $G$ is a (unique) path.

Construction 5. Let $\lambda \geq 2$ be an integer. We construct a neutral connector gadget composed of a graph $G_{\text {neutral }}=\left(V_{\text {neutral }}, E_{\text {neutral }}\right)$ and obligations $\Pi_{\text {neutral }}$ as follows:

- We add a path $Q_{1}, \ldots, Q_{3 \lambda}$.

- We add three obligations $V_{1}, V_{2}$ and $V_{3}$.

- We add $Q_{1}, Q_{3}, Q_{3 \lambda-3}$ in $V_{1}, Q_{2}, Q_{3 \lambda-1}$ in $V_{2}$ and $Q_{4}, Q_{3 \lambda-2}, Q_{3 \lambda}$ in $V_{3}$. 
- For each vertex $Q_{i} \in\left\{Q_{5}, \ldots, Q_{3 \lambda-4}\right\}$ :

- If $i(\bmod 3)=0$, we add $Q_{i}$ in $V_{1}$.

- If $i(\bmod 3)=2$, we add $Q_{i}$ in $V_{2}$.

- If $i(\bmod 3)=1$, we add $Q_{i}$ in $V_{3}$.
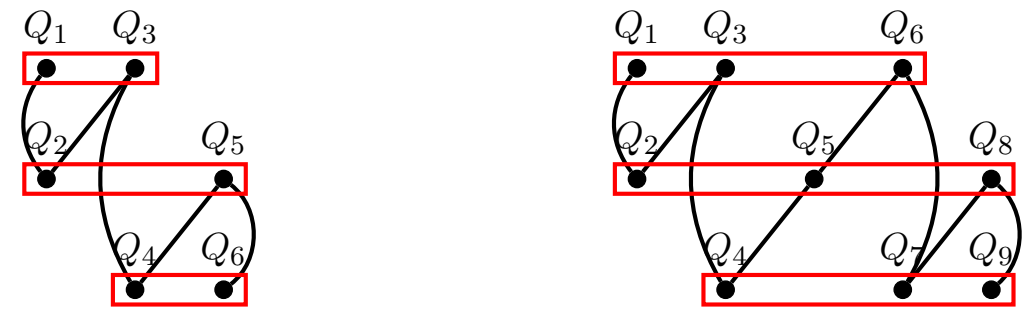

Figure 6: A neutral connector following Construction 5 where $\lambda=2$ on the left and $\lambda=3$ on the right.

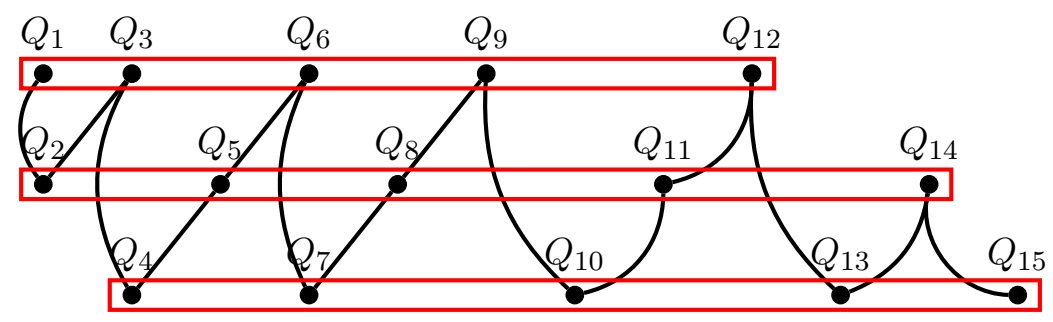

Figure 7: A neutral connector following Construction 5 where $\lambda=5$.

Note that $G_{\text {neutral }}$ is a (unique) path, with three stable $\lambda$-balanced obligations. See illustrations in Figure 6 and Figure 7. We now show that this gadget is a neutral connector for the $I D O$ problem, i.e. it can be added to an initial instance without changing the property of having or not an $I D O$.

It is easy to show that the only IDO of $\left(G_{\text {neutral }}, \Pi_{\text {neutral }}\right)$ is obligation $V_{2}$.

To show the neutrality, let us consider any instance $I=(G, \Pi)$ of the $I D O$ problem and add the neutral gadget $\left(G_{\text {neutral }}, \Pi_{\text {neutral }}\right)$ (for any value of $\lambda$ ) to it as follows. Connect with a new edge any vertex of the graph $G$ of $I$ and the first vertex $Q_{1}$ of the path $G_{\text {neutral }}$; also connect with a new edge any other vertex of the graph $G$ of $I$ and the other extremity $Q_{3 \lambda}$ of the path $G_{n e u t r a l}$. Let $I^{\prime}$ be this new instance of the IDO problem.

Suppose that the initial instance $I$ has an IDO $S$. Then clearly $S \cup V_{2}$ is an $I D O$ of instance $I^{\prime}$.

Suppose now that $I^{\prime}$ has an IDO S. $S$ contains $V_{2}$ but not obligations $V_{1}$ or $V_{3}$ (otherwise some vertices of $G_{\text {neutral }}$ would not be dominated by $S$ or $S$ would not be an independent set). This means that no vertex of $G_{\text {neutral }}$ can help to dominate vertices of graph $G$ of $I$. Hence, the obligations of $I$ included in $S$ form an IDO of $I$. 
Now to get the $N P$-completeness for a (unique) path and stable and $\lambda$-balanced obligations, where $\lambda \geq 2$ is a constant integer, we connect with neutral connector gadgets all the disjoint paths composing the graph $G$ obtained after Construction 4. For that, number (in any order) the $l$ disjoint paths composing $G: P_{1}, \ldots, P_{l}$. We note $a_{i}$ and $b_{i}$ the two extremities of path $P_{i}$. Now for each $i=1, \ldots, l-1$ construct a new neutral network gadget $\lambda$-balanced and connect $b_{i}$ to $Q_{1}$ and $Q_{3 \lambda}$ to $a_{i+1}$.

Based on the neutrality of the neutral connector gadgets, it is possible to complete the proof of Theorem 2 to get the following theorem.

Theorem 3. The IDO problem is NP-complete, even if the graph is a (unique) path and the obligations are stable and $\lambda$-balanced, where $\lambda \geq 2$ is a constant integer.

In the rest of this section, we show how to obtain other $N P$-completeness results in various other types of instances. For example, we can prove that the IDO problem is $N P$-complete even if the graph has a low (resp. high) density of edges. For that, just add new complete graphs (resp. independent vertices) with singleton obligations to the previous initial construction. The $N P$-completeness is kept for these new instances. We do not give more details here.

We focus on the distances in the graph and study their potential impact on complexity of the IDO problem. The distance between two vertices $u$ and $v$ in a graph is the length of the shortest path between $u$ and $v$. The diameter of a graph is the largest distance between its vertices. We now show that the IDO problem is $N P$-complete even in a graph of diameter three with stable and $\lambda$-balanced obligations, where $\lambda \geq 2$ is a constant integer.

The general idea is the following. First, we construct an instance $(G, \Pi)$ of $I D O$ with Construction 4. At this step, we have a unique path and many $\lambda$-balanced obligations. To reduce the diameter of this path, we add a new obligation $\Lambda$ with $\lambda$ vertices. Each vertex of $\Lambda$ is a universal vertex of $G$; i.e., each vertex of $\Lambda$ is adjacent to all other vertices of the graph $G$. The instance has a graph of diameter two and still has $\lambda$-balanced obligations. The last step is to prevent these new vertices from being in an IDO. We could add an edge between two vertices of $\Lambda$, but this obligation would no longer be stable. Instead, we will use a property of neutral connectors. Besides connecting two graphs without affecting the existence of an IDO, a neutral connector admits only one IDO. If the obligation containing $Q_{2}$ and $Q_{3 \lambda-1}$ is not in an $I D O$, either $Q_{3}$ or $Q_{3 \lambda-2}$ cannot be dominated. Thus we add a new neutral connector $N_{c}$ of size $3 \lambda$. Each vertex of $\Lambda$ is adjacent to each vertex of $N_{c}$ except $Q_{3}$. An IDO must have the vertices of the obligation containing $Q_{2}$ and $Q_{3 \lambda-1}$. The vertices of $\Lambda$ can no longer be in an IDO.

Construction 6. Let $(\mathcal{X}, \mathcal{C})$ be an instance of $R X 3 C$ where $\mathcal{X}=\left\{x_{1}, \ldots, x_{3 q}\right\}$ and $\mathcal{C}=$ $\left\{c_{1}, \ldots, c_{3 q}\right\}$. Let $\lambda \geq 2$ be an integer. We construct an IDO instance $(G=(V, E), \Pi)$ as follows:

- We follow Construction 4. 
- We add one neutral connector $N_{c}$ following Construction 5 with obligations of size $\lambda$.

- We add $\lambda$ new vertices all contained in one obligation $\Lambda=\left\{v_{1}, \ldots, v_{\lambda}\right\}$.

- We add all edges between each vertex $v_{i}$ of $\left\{v_{1}, \ldots, v_{\lambda}\right\}$ and all the vertices of the other vertices, except vertex $Q_{3}$ of $N_{c}$.

Note that $G$ is a graph of diameter three and $\Pi$ contains $68 q$ stable $\lambda$-balanced obligations. See an illustration in Figure 8. The edges between vertices of $\Lambda$ and vertices of the neutral connector, as well as the edges between vertices of the neutral connector, have different colors for clarity purposes. The dashed edges symbolize the edges between each vertex of $G \backslash\left\{v_{1}, \ldots, v_{\lambda}, Q_{1}, \ldots, Q_{3 \lambda}\right\}$ and each vertex of $\Lambda$.

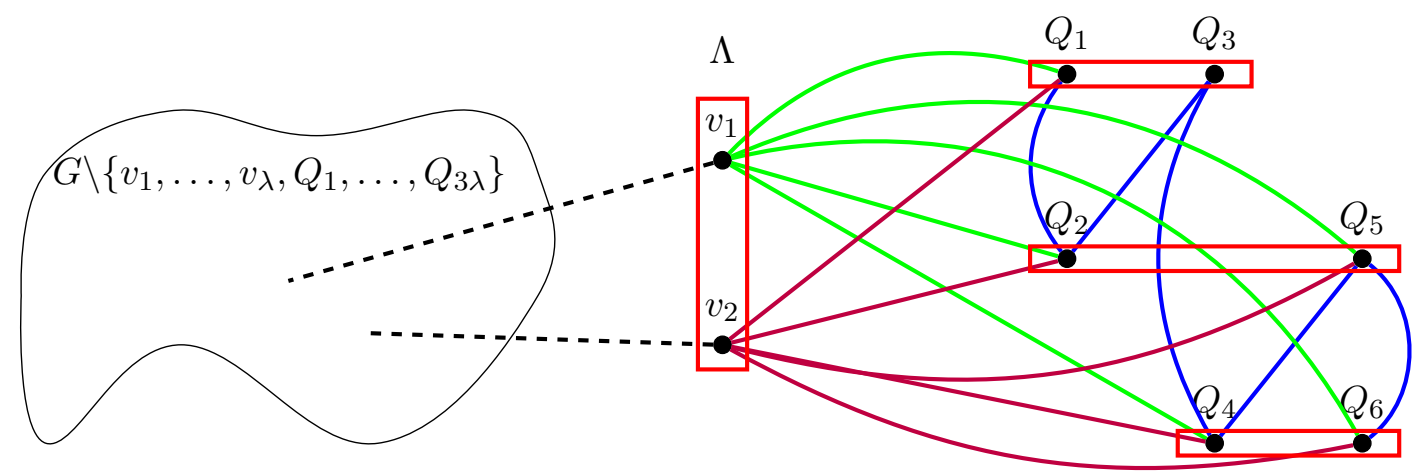

Figure 8: A graph following Construction 6 where $\lambda=2$.

Theorem 4. The IDO problem is NP-complete, even if the graph is of diameter three and the obligations are stable and $\lambda$-balanced, where $\lambda \geq 2$ is a constant integer.

Proof. Each vertex of $V \backslash\left(Q_{3} \cup \Lambda\right)$ is at distance one to vertices of $\Lambda$. Each vertex of $\Lambda$ is at distance two to other vertices of $\Lambda$. The vertex $Q_{3}$ is at distance two to all the vertices of $\Lambda$. Thus, the graph has a diameter three.

The vertices $\left\{v_{1}, \ldots, v_{\lambda}\right\}$ cannot be in an IDO since it would be the only obligation in it and in this case vertex $Q_{3}$ of the neutral connector is not dominated. The only possible $I D O$ is composed of an IDO of the initial instance constructed by Construction 4 plus obligation $V_{2}$ of the neutral connector. Hence, the new instance has an IDO if and only if the initial instance has an IDO.

We have shown in this section that the IDO problem is $N P$-complete even in the very restricted case where the graph is a path, and the obligations are stable $\lambda$-balanced where $\lambda \geq 2$ is a constant. In the next section, we will study the complexity of the problem when the size of obligations are non-constant. 


\section{Instances with balanced non constant size obligations}

In this section we first propose another polynomial reduction between $R X 3 C$ and $I D O$ problems. The rest of the section mainly consists in exploiting variants of this initial construction. This leads us to show that the IDO problem is $N P$-complete even if the number of balanced obligations is equal to (resp. lower than, resp. higher than) the square root of the number of vertices of the graph.

Construction 7. Let $(\mathcal{X}, \mathcal{C})$ be an instance of $R X 3 C$ where $\mathcal{X}=\left\{x_{1}, \ldots, x_{3 q}\right\}$ et $\mathcal{C}=$ $\left\{c_{1}, \ldots, c_{3 q}\right\}$. We construct a graph $G=(V, E)$ and obligations $\Pi$ as follows:

- For each $x_{i} \in X$, we add the vertex $x_{i}$.

- For each $c_{i} \in C$, we add the vertex $z_{i}$.

- For each $c_{i} \neq c_{j} \in \mathcal{C}$, if $c_{i}$ and $c_{j}$ share at least one element then we add the edge $\left(z_{i} z_{j}\right)$ (the two vertices representing two non disjoint triplets are linked).

- For each $c_{i}=\left\{x_{j}, x_{k}, x_{l}\right\} \in C$, we add the edges $\left(z_{i} x_{j}\right),\left(z_{i} x_{k}\right)$ and $\left(z_{i} x_{l}\right)$.

- We add the vertices $y_{1}, y_{2}$ and $y_{3}$, and the edge $\left(y_{1} y_{2}\right)$.

- We add the obligations $\left\{x_{1}, \ldots, x_{3 q}, y_{1}\right\}$ and $\left\{y_{2}, y_{3}\right\}$.

- Each other vertex is in an obligation of size one.

Note that $G$ is a graph with $6 q+3$ vertices and $\Pi$ has $3 q+2$ stable non balanced obligations. This instance can be constructed in polynomial time from $(\mathcal{X}, \mathcal{C})$. See an illustration in Figure 9.

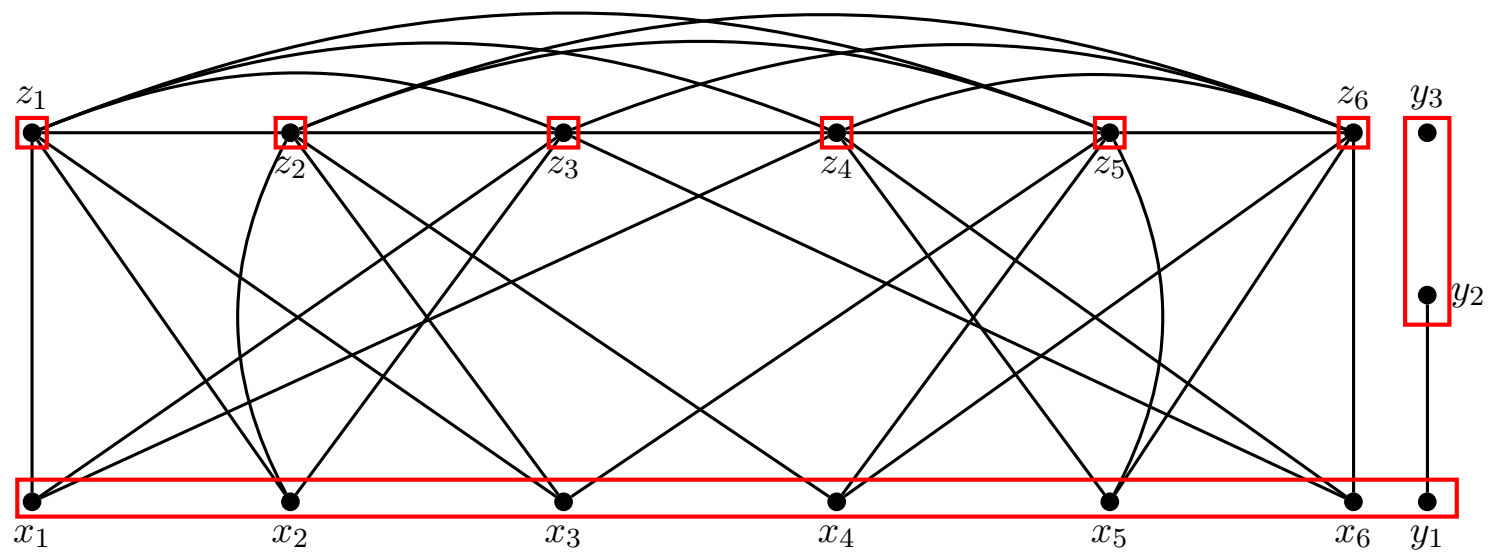

Figure 9: Illustration of Construction 7 for the instance $(\mathcal{X}, \mathcal{C})$ of $R X 3 C$ where $\mathcal{X}=\{1, \ldots, 6\}$ and $\mathcal{C}=\{\{1,2,3\},\{2,3,4\},\{1,2,6\},\{1,5,6\},\{3,4,5\},\{4,5,6\}\}$. 
Lemma 5. Let $(\mathcal{X}, \mathcal{C})$ be an instance of $R X 3 C$. Instance $(\mathcal{X}, \mathcal{C})$ has a solution if and only if the instance $(G, \Pi)$ constructed from $(\mathcal{X}, \mathcal{C})$ following Construction 7 contains an IDO.

Proof. Suppose there is a solution $S$ for the instance $(\mathcal{X}, \mathcal{C})$ of $R X 3 C$, where $\mathcal{X}=$ $\left\{x_{1}, \ldots, x_{3 q}\right\}$ and $\mathcal{C}=\left\{c_{1}, \ldots, c_{3 q}\right\}$. Let us construct $S^{\prime}$ a solution for the instance $(G, \Pi)$ following Construction 7. For each $c_{i} \in S$, we put $z_{i} \in S^{\prime}$. We also put the vertices $y_{2}$ and $y_{3}$ in $S^{\prime}$. Set $S^{\prime}$ is then a stable set of $G$. Now, as $S$ covers all elements of $\mathcal{X}$, all of the $z$-vertices and the $x$-vertices are dominated by $S^{\prime}$. Thus $S^{\prime}$ is an IDO.

Suppose now that there is an IDO $S$ for the instance $(G, \Pi)$ from Construction 7 . Let us construct $S^{\prime}$ a solution for the instance $(\mathcal{X}, \mathcal{C})$ of $R X 3 C$ as follows: For each $z_{i} \in S$, we put $c_{i} \in S^{\prime}$. Note that vertex $y_{3}$ can only be dominated by itself. Thus obligation $\left\{y_{2}, y_{3}\right\}$ is in $S$ and then no $x$-vertex can be in $S$, so they are dominated by $z$-vertices. Since $S$ is an independent set, each $x$-vertex is dominated by exactly one $z$-vertex of $S$. Thus, $S^{\prime}$ is a solution for $R X 3 C$.

We now propose modifications of Construction 7 to show the $N P$-completeness on instances with $\sqrt{N}$ stable and $\sqrt{N}$-balanced obligations, where $N$ is the number of vertices of the graph.

Construction 8. Let $(\mathcal{X}, \mathcal{C})$ be an instance of $R X 3 C$ where $\mathcal{X}=\left\{x_{1}, \ldots, x_{3 q}\right\}$ and $\mathcal{C}=$ $\left\{c_{1}, \ldots, c_{3 q}\right\}$. We construct a graph $G=(V, E)$ and obligations $\Pi$ as follows:

- We use Construction 7 to get an initial instance.

- We add new vertices $y^{4} \ldots, y^{3 q+3}$ to the obligation which contains $\left\{y_{2}, y_{3}\right\}$.

- We add the new vertex $y_{1}^{\prime}$ to the obligation which contains $y_{1}$ and we add the edge $\left(y_{1}^{\prime} y_{2}\right)$ in the graph.

- For each $z_{i} \in V$, we add the new vertices $z_{i}^{1}, \ldots, z_{i}^{3 q+1}$ in the obligation which contains only $z_{i}$.

- For each pair of vertices $z_{i} \neq z_{j}$ of the initial instance, we add the edges $\left(z_{i}^{1} z_{j}\right), \ldots,\left(z_{i}^{3 q+1} z_{j}\right)$ and $\left(z_{j}^{1} z_{i}\right), \ldots,\left(z_{j}^{3 q+1} z_{i}\right)$ if and only if the edge $\left(z_{i} z_{j}\right)$ is in the initial instance.

Note that $G$ has $N=9 q^{2}+12 q+4$ vertices, and the $3 q+2=\sqrt{N}$ obligations are all independent and all of size $3 q+2=\sqrt{N}$. Since the transformations of Construction 7 do not affect the existence of a solution, compared to the initial instance, the proof of Lemma 5 is still valid for Construction 8 and can be used to prove the following result.

Theorem 5. The IDO problem is NP-complete even if there are $\sqrt{N}$ stable and $\sqrt{N}$ balanced obligations, where $N$ is the number of vertices of the graph. 
With Construction 8 we have constructed instances of the IDO problem with $\sqrt{N}$ stable and $\sqrt{N}$-balanced obligations. Now we explore two other cases. Firstly let us see the case of instances with strictly more than $\sqrt{N}$ balanced obligations. We take an initial instance obtained by Construction 8 and note $q$ the size of the obligations (they are all the same). Then we add to this instance $p q$ new vertices, but no edges. With these $p q$ vertices we arbitrarily create $p$ new disjoint stable obligations, all of size $q$. Let $N$ be the number of vertices of this new graph. This new instance contains strictly more than $\sqrt{N}$ balanced obligations. However, the new instance has an IDO if and only if the initial instance contains an IDO. Using the result of Theorem 5 leads to the next result.

Corollary 1. The IDO problem is NP-complete even if there are at least $\sqrt{N}$ (and less than $N$ ) stable and balanced obligations, where $N$ is the number of vertices of the graph.

Now let us see the case of balanced obligations with more than $\sqrt{N}$ vertices in each obligation (thus leading to strictly less than $\sqrt{N}$ obligations). We take Construction 8 and add a new vertex in each obligation. The new vertex in the same obligation than $y_{3}$ (resp. $y_{1}$ ) is not connected (resp. connected to $y_{2}$ ). The new vertex added in the obligation containing $z_{i}$ is connected to the same neighbours than the other vertices $z_{i}^{j}$ of this obligation. Then this newly defined instance has strictly more than $\sqrt{N}$ vertices in each balanced obligation. We can continue to add vertices in each obligation with the same procedure, without changing the property of having or not an IDO compared to the initial instance. This construction leads to the following corollary.

Corollary 2. The IDO problem is NP-complete even if there are at most $\sqrt{N}$ stable and balanced obligations, where $N$ is the number of vertices of the graph.

Note that when the number \#o of obligations is constant (independent of $N$ ), the IDO problem is polynomial. Indeed, an algorithm can explore all the possible $O\left(2^{\# o}\right)$ combinations of obligations in polynomial time.

\section{Maximising the number of dominated vertices}

Since determining if there is an $I D O$ is $N P$-complete, even in a very restricted family of instances, in this section we relax the constraint of dominating all the vertices of the graph.

\section{Partially Independent Dominating set with Obligation (PIDO).}

Given an instance composed of a graph $G=(V, E)$, and obligations $\Pi=\left\{V_{1}, \ldots, V_{k}\right\}$, a set of vertices $S \in V$ is a PIDO if $S$ is an independent set of graph $G$ and respects the obligations of $\Pi$.

To define a measure associated to a PIDO $S$, let $N(S)$ be the set of vertices of $G$ that have at least one neighbour in (i.e. are dominated by) $S$. Then, the number of vertices dominated by a PIDO $S$ is defined by $|S \cup N(S)|$. 
An $I D O S$ of an instance $(G=(V, E), \Pi)$ is then a $P I D O$ of instance $(G=(V, E), \Pi)$ dominating all the $|V|$ vertices of $G$. To ensure that there is always a non-empty PIDO, we will only consider instances with stable obligations. Note that in this case, any (stable) obligation is a PIDO dominating at least its own vertices.

The associated decision problem is the following.

\section{PIDO Problem:}

Instance: $G=(V, E)$ an undirected graph, $\Pi=V_{1}, \ldots, V_{k}$ a partition of $V$ and an integer $K$.

Question: Does $(G, \Pi)$ contain a PIDO dominating at least $K$ vertices of $G$ ?

In this section, we show that the PIDO problem is $N P$-complete, when $K=3 \sqrt{|V|}-2$ even if the graph is a collection of disjoint paths and the obligations are stable. To obtain this result we do a reduction from the $I D O$ problem. As a second step we will propose a polynomial time algorithm to construct a PIDO dominating at least $2 \sqrt{|V|}-1$ vertices in any instance $(G=(V, E), \Pi)$ if the obligations of $\Pi$ are stable.

The first step of the proof of $N P$-completeness is to construct a gadget that allows us to limit the potential of domination.

Construction 9. Let $Y$ be an integer. We construct $G_{Y}=\left(V_{Y}, E_{Y}\right)$ a graph and obligations $\Pi_{Y}$ as follows:

- We add the vertices $v_{1}^{1}, \ldots, v_{Y}^{1}, \ldots, v_{1}^{Y}, \ldots, v_{Y}^{Y}$.

- We add the obligations $\left\{v_{1}^{1}, \ldots, v_{1}^{Y}\right\}, \ldots,\left\{v_{Y}^{1}, \ldots, v_{Y}^{Y}\right\}$.

- For each $1 \leq i \neq j \leq Y$, we add the edge $\left(v_{i}^{j} v_{j}^{i}\right)$.

- We delete the vertices $v_{1}^{1}, v_{2}^{2}, \ldots, v_{Y-1}^{Y-1}, v_{Y}^{Y}$.

Note that $G_{Y}$ is a collection of disjoint paths with $Y^{2}-Y$ vertices and $Y$ stable obligations, each of size $Y-1$. See an illustration in Figure 10.

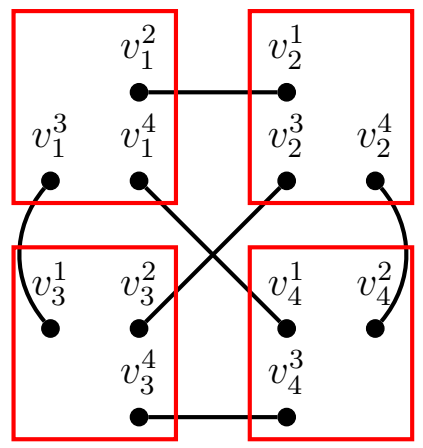

Figure 10: Illustration of Construction 9 for $Y=4$. 
Lemma 6. Let $\left(G_{Y}, \Pi_{Y}\right)$ be an instance constructed following Construction 9. For any value of $Y \geq 2$, any PIDO cannot dominate more than $2 Y-2$ vertices in it.

Proof. Since a PIDO must respect the obligations, let us consider any obligation $V_{i} \in \Pi_{Y}$. Let $V_{i}=\left\{v_{i}^{1}, \ldots, v_{i}^{Y}\right\}$. Suppose that a PIDO $S$ contains it: $\left\{v_{i}^{1}, \ldots, v_{i}^{Y}\right\} \subseteq S$. Because of the construction, an edge connects $V_{i}$ and all the other obligations. Thus, no more vertices can be added to $S$. Each vertex of $S$ dominates itself and another vertex in another obligation i.e. $S$ dominates exactly $2 Y-2$ vertices. Hence any PIDO can dominate $2 Y-2$ vertices but no more.

As previously mentioned, to prove our $N P$-completeness result, we do not make a reduction from $R X 3 C$ but from IDO problem which is NP-Complete (see section 2). The overall strategy of the reduction here is to consider an instance of the IDO problem and then to add the previous gadget $\left(G_{Y}, \Pi_{Y}\right)$ with a well-chosen parameter $Y$.

Construction 10. Let $(G, \Pi)$ be an instance of IDO where $G=(V, E)$ is a path of vertices and $\Pi=\left\{V_{1}, \ldots, V_{l}\right\}$ are stable obligations. We construct a graph $G^{\prime}=\left(V^{\prime}, E^{\prime}\right)$ and obligations $\Pi^{\prime}$ as follows:

- Let $\left(G_{Y}, \Pi_{Y}\right)$ be an instance of IDO following Construction 9 where $Y=|V|$.

- $G^{\prime}$ is the union of the two disjoint graphs $G$ and $G_{Y}$ and $\Pi^{\prime}=\Pi \cup \Pi_{Y}$.

Note that $G^{\prime}$ is a collection of disjoint paths with $|V|^{2}$ vertices, and $\Pi^{\prime}$ contains stable obligations. This new instance can be constructed in polynomial time from the initial instance $(G=(V, E), \Pi)$.

Theorem 6. Determining if there is a solution for the PIDO problem which can dominate at least $3 \sqrt{N}-2$ vertices (where $N$ is the number of vertices of the graph) is NP-complete even if the graph is a collection of disjoint paths and the obligations are stable.

Proof. The problem is clearly in NP. We have shown in Theorem 3 that the IDO problem is $N P$-complete in instance $(G, \Pi)$ even if $G=(V, E)$ is a path and $\Pi$ are stable obligations. We make the reduction from that. We note $\left(G^{\prime}=\left(V^{\prime}, E^{\prime}\right), \Pi^{\prime}\right)$ the instance obtained (in polynomial time) from $(G, \Pi)$ by Construction 10. By construction, we have $\left|V^{\prime}\right|=|V|^{2}$.

Suppose there is an IDO $S$ for the instance $(G, \Pi)$ of the IDO problem. Let us construct $S^{\prime}$ a $P I D O$ for the instance $\left(G^{\prime}, \Pi^{\prime}\right)$. For that, simply take in $S^{\prime}$ all the vertices of $S$ and add one $Y$-obligation. Set $S^{\prime}$ is an independent set of $G^{\prime}$ and dominates all the $|V|$ vertices of the " $G$ part" of $G^{\prime}$ plus $2|V|-2$ vertices in the gadget, i.e. at least $3 \sqrt{\left|V^{\prime}\right|}-2$ vertices of $G^{\prime}$.

Suppose now that there is a PIDO $S$ for the PIDO instance $\left(G^{\prime}, \Pi^{\prime}\right)$, dominating at least $3 \sqrt{\left|V^{\prime}\right|}-2$ vertices of $G^{\prime}$. Let us construct $S^{\prime}$ a solution for the instance $(G, \Pi)$ of IDO. Since $S^{\prime}$ can only dominate $2 \sqrt{\left|V^{\prime}\right|}-2$ vertices of the gadget part $\left(G_{Y}\right)$ of $G^{\prime}, S^{\prime}$ must dominate all the $|V|$ vertices of the other part $G=(V, E)$. Thus there is a solution for the $I D O$ problem in $G$. 
We have shown that determining if there is a solution for the PIDO problem which can dominate at least $3 \sqrt{N}-2$ vertices (where $N$ is the number of vertices of the graph) is $N P$-complete even if the graph is a collection of disjoint paths and the obligations are stable. On the other side, we show now that if the obligations are stable, an instance $(G=(V, E), \Pi)$ always contains a PIDO dominating at least $2 \sqrt{|V|}-1$ vertices. Moreover, such a solution can be constructed in polynomial time. We also show that the bound of $2 \sqrt{|V|}-1$ is tight.

Consider the following greedy algorithm for any instance $(G, \Pi)$ with stable obligations.

1. Create an initial empty solution $S$.

2. While there are obligations, do:

(a) Add the vertices of the biggest obligation $B$ in $S$.

(b) Let $N(B)$ be the set of vertices that are neighbour of at least a vertex of $B$. Let $O(B)$ be the set of all vertices contained in obligations containing at least a vertex of set $N(B)$ (i.e. $O(B)$ contains all the vertices of all obligations that are "neighbour" of $B$ ). From the current instance, delete all the vertices contained in obligation $B$ plus all the vertices of $O(B)$ (and all the corresponding obligations).

\section{Return $S$.}

Since the number of obligations strictly decreases at each step of the loop, the algorithm terminates. Note that, as obligations are stable, $S$ is non empty and, by greedy construction, dominates at least one vertex of every obligation. Due to the update process of the instance step by step, $S$ respects the obligations and is an independent set of the graph $G=(V, E)$. As there are at most $|V|$ obligations, the algorithm is polynomial.

Lemma 7. Let $(G=(V, E), \Pi)$ be any instance with stables obligations. This instance always has a PIDO dominating at least $2 \sqrt{|V|}-1$ vertices that can be constructed by the greedy polynomial algorithm described above.

Proof. Let $(G=(V, E), \Pi)$ be an instance of PIDO, with stable obligations and $n=|V|$ be the number of vertices of the graph. Let \#o be the number of obligations in $\Pi$ and $|o|_{\max }$ the size of the biggest obligation of $\Pi$. The greedy algorithm described above returns a stable set of vertices, respecting obligations and dominating at least $\# o+|o|_{\max }-1$ vertices. As we have the trivial inequality $\# o \times|o|_{\max } \geq n$, we have $\# o \geq \sqrt{n}$ or $|o|_{\max } \geq \sqrt{n}$. W.l.o.g. suppose that $|o|_{\max } \geq \sqrt{n}$. We can rewrite this inequality as $|o|_{\max }=\sqrt{n}+x$, with $x>0$. Suppose now by contradiction that $\# o+|o|_{\max }<2 \sqrt{n}$ :

$$
\begin{aligned}
\# o+|o|_{\max } & <2 \sqrt{n} \\
\# o+\sqrt{n}+x & <2 \sqrt{n} \\
\# o & <\sqrt{n}-x
\end{aligned}
$$


With this inequality we get: $\# o \times|o|_{\max } \leq(\sqrt{n}-x)(\sqrt{n}+x) \leq n-x^{2}$. But, as $\# o \times|o|_{\max } \geq n$ we get a contradiction. Thus we have $\# o+|o|_{\max } \geq 2 \sqrt{n}$, and so $\# o+|o|_{\max }-1 \geq 2 \sqrt{n}-1$.

We now show that this bound of $2 \sqrt{|V|}-1$ is tight by using the following instances.

Construction 11. Let $Y$ be an integer. We construct $G=(V, E)$ a graph and $\Pi$ obligations $\Pi$ as follows:

- We add the vertices $v_{1}^{1}, \ldots, v_{Y}^{1}, \ldots, v_{1}^{Y}, \ldots, v_{Y}^{Y}$.

- We add the obligations $\left\{v_{1}^{1}, \ldots, v_{1}^{Y}\right\}, \ldots,\left\{v_{Y}^{1}, \ldots, v_{Y}^{Y}\right\}$.

- For each $1 \leq i \neq j \leq Y$, we add the edge $\left(v_{i}^{j} v_{j}^{i}\right)$.

Note that $G$ contains $Y^{2}$ vertices and $\Pi$ is composed of $Y$ stable and $Y$-balanced obligations. See an illustration in Figure 11.

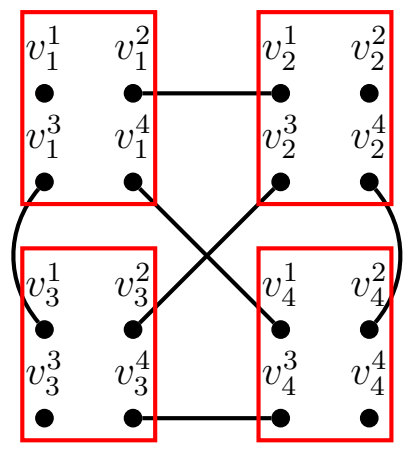

Figure 11: Illustration of Construction 11 for $Y=4$.

Lemma 8. Any instance $(G=(V, E), \Pi)$ obtained by Construction 11 with any parameter $Y \geq 2$ does not contain a PIDO dominating more than $2 \sqrt{|V|}-1$ vertices.

Proof. Let $(G=(V, E), \Pi)$ be obtained by Construction 11. Since each obligation shares exactly one edge with each other obligation, only one obligation can be in a PIDO. As each obligation has $\sqrt{|V|}$ vertices and as there are $\sqrt{|V|}$ obligations, any non-empty PIDO dominates exactly $2 \sqrt{|V|}-1$ vertices, regardless of the chosen obligation.

We have shown that there is always a PIDO dominating $2 \sqrt{N}-1$ vertices (where $N$ is the number of vertices of the graph) if the obligations are stable. We have also shown that there are instances in which no more than $2 \sqrt{N}-1$ vertices can be dominated. Thus we can conclude this section by the following theorem.

Theorem 7. Let $(G=(V, E), \Pi)$ be an instance with stable obligations. It contains a PIDO dominating at least $2 \sqrt{|V|}-1$ vertices (this bound is tight for an infinite number of instances). Such a PIDO can be constructed in polynomial time. 


\section{Conclusion}

In this paper, we have shown that the IDO problem is $N P$-complete, even if the graph is a path and the obligations are stable and $\lambda$-balanced (where $\lambda \geq 2$ is a constant integer), or if the graph has a diameter three and the obligations are stable and $\lambda$-balanced (with $\lambda \geq 2$ ). However, determining if there is a solution for the IDO problem is clearly polynomial for graphs with diameter one. The case of diameter two is still open. We have also shown that the IDO problem is NP-complete, even if there are less or more than $\sqrt{N}$ stable balanced obligations. Finally, we introduced a version of the problem where the goal is to dominate the maximum number of vertices while respecting the obligations. We proved that it is NP-complete to determine if it is possible to dominate only at least $3 \sqrt{N}-2$ vertices (where $N$ is the number of vertices of the graph). However, we proved that there is always a solution which can dominate at least $2 \sqrt{N}-1$ vertices if obligations are stable. We have also given a polynomial algorithm to construct such a solution. However, reducing the small gap between polynomial and NP-complete cases is still open.

\section{References}

[1] A. Akbari et al. Independent Domination in Subcubic Graphs. 2020. arXiv: 2001. 02946 [cs.DM].

[2] Claude Berge. "Theory of Graphs and its Applications". In: IL, Moscow (1962), pp. $150-152$.

[3] Miroslav Chlebík and Janka Chlebíková. "Approximation Hardness of Dominating Set Problems". In: Algorithms - ESA 2004. Ed. by Susanne Albers and Tomasz Radzik. Berlin, Heidelberg: Springer Berlin Heidelberg, 2004, pp. 192-203.

[4] Alexis Cornet and Christian Laforest. "Domination problems with no conflicts". In: Discret. Appl. Math. 244 (2018), pp. 78-88.

[5] Alexis Cornet and Christian Laforest. "Graph Problems with Obligations". In: Combinatorial Optimization and Applications. Ed. by Donghyun Kim, R. N. Uma, and Alexander Zelikovsky. Vol. (LNCS) 11346. Cham: Springer International Publishing, 2018, pp. 183-197.

[6] Alexis Cornet and Christian Laforest. "Total Domination, Connected Vertex Cover and Steiner Tree with Conflicts". In: Discret. Math. Theor. Comput. Sci. 19.3 (2017).

[7] Michael R. Garey and David S. Johnson. Computers and Intractability: A Guide to the Theory of NP-Completeness. New York, NY, USA: W. H. Freeman \& Co., 1979.

[8] Teresa W Haynes, Stephen Hedetniemi, and Peter Slater. Domination in Graphs: Advanced Topics. CRC press, 1998.

[9] Teresa W Haynes, Stephen Hedetniemi, and Peter Slater. Fundamentals of domination in graphs. Marcel Dekker, 1998. 
[10] Robert W. Irving. "On approximating the minimum independent dominating set". In: Information Processing Letters 37.4 (1991), pp. 197-200.

[11] Mamadou Moustapha Kanté, Christian Laforest, and Benjamin Momège. "An Exact Algorithm to Check the Existence of (Elementary) Paths and a Generalisation of the Cut Problem in Graphs with Forbidden Transitions". In: SOFSEM 2013 Proceedings. Ed. by Peter van Emde Boas et al. Vol. 7741. Lecture Notes in Computer Science. Springer, 2013, pp. 257-267.

[12] Mamadou Moustapha Kanté, Christian Laforest, and Benjamin Momège. "Trees in Graphs with Conflict Edges or Forbidden Transitions". In: Theory and Applications of Models of Computation, 10th International Conference, TAMC 2013, Hong Kong, China, May 20-22, 2013. Proceedings. Ed. by T.-H. Hubert Chan, Lap Chi Lau, and Luca Trevisan. Vol. 7876. Lecture Notes in Computer Science. Springer, 2013, pp. 343-354.

[13] Mamadou Moustapha Kanté et al. "Finding Paths in Grids with Forbidden Transitions". In: 41st International Workshop, WG 2015. Ed. by Ernst W. Mayr. Vol. 9224. Lecture Notes in Computer Science. Springer, 2015, pp. 154-168.

[14] Christian Laforest and Benjamin Momège. "Nash-Williams-type and Chvátal-type Conditions in One-Conflict Graphs". In: SOFSEM 2015 Proceedings. Ed. by Giuseppe F. Italiano et al. Vol. 8939. Lecture Notes in Computer Science. Springer, 2015, pp. 327-338.

[15] Christian Laforest and Benjamin Momège. "Some Hamiltonian Properties of OneConflict Graphs". In: 25th International Workshop, IWOCA 2014. Ed. by Jan Kratochvíl, Mirka Miller, and Dalibor Froncek. Vol. 8986. Lecture Notes in Computer Science. Springer, 2014, pp. 262-273.

[16] Oystein Ore. Theory of graphs. Vol. 38. American Mathematical Soc., 1965.

[17] Johan M.M. [van Rooij] and Hans L. Bodlaender. "Exact algorithms for dominating set". In: Discrete Applied Mathematics 159.17 (2011), pp. 2147-2164. 